\title{
Severe Gastrointestinal Involvement in Pediatric Stevens-Johnson Syndrome: A Case Report and Review of the Literature
}

This article was published in the following Dove Press journal:

Clinical and Experimental Gastroenterology

\author{
Sophia Bechek' \\ Manuel Garcia ${ }^{2}$ \\ Howard Chiou ${ }^{3}$ \\ 'Stanford University School of Medicine, \\ Stanford, CA, USA; '2Division of Pediatric \\ Gastroenterology, Stanford University \\ School of Medicine, Stanford, CA, USA; \\ ${ }^{3}$ Department of Medicine, Santa Clara \\ Valley Medical Center, San Jose, CA, USA
}

\begin{abstract}
Stevens-Johnson syndrome and toxic epidermal necrolysis form a rare but severe disease spectrum characterized by widespread epidermal detachment. Gastrointestinal manifestations of the disease, however, are rarely described in the pediatric literature and have a high mortality among adults. There are limited data on the treatment of these cases, with conflicting evidence regarding the benefit of steroids, IVIG, or other immunosuppressive agents. We review previous instances of gastrointestinal involvement in children and report the case of a previously healthy 13-year-old who presented with the typical ocular and skin findings of Stevens-Johnson syndrome, subsequently developed severe life-threatening diarrhea, and was found to have severe esophagitis, duodenitis, and colitis on endoscopic evaluation. Treatment was initiated with an immediate, short course of steroids along with early introduction of an enteral diet via nasogastric tube, and resulted in full gastrointestinal recovery. This case highlights successful medical treatment of the first reported pediatric case of SJS/TEN with both upper and lower gastrointestinal tract involvement.
\end{abstract}

Keywords: Stevens-Johnson syndrome, gastrointestinal involvement, diarrhea, steroids, enteral nutrition

\section{Introduction}

Stevens-Johnson syndrome (SJS) and toxic epidermal necrolysis (TEN) form a rare but severe disease spectrum characterized by a hypersensitivity reaction that leads to varied degrees of epidermal detachment. The disease often leads to sepsis and multi-organ failure, resulting in $16 \%$ mortality among children when more than $30 \%$ of the body surface is involved. ${ }^{1}$ Widespread gastrointestinal involvement in $\mathrm{SJS} / \mathrm{TEN}$, however, is very rarely reported in the pediatric literature and the number of reported cases is insufficient to accurately predict prognosis or standardize management. Among adults, gastrointestinal manifestations are a marker of disease severity with an estimated mortality of $52 \%$ among cases with involvement beyond the esophagus. ${ }^{2-15}$

We present the case of a boy who presented with the typical cutaneous and ocular manifestations of SJS, and subsequently developed severe diarrhea with diffuse involvement of the esophagus, small bowel, and colon. This patient is the first reported pediatric case of SJS/TEN in which both the upper and lower gastrointestinal tracts are involved. The case highlights the importance of providers recognizing manifestations of this disease beyond the skin and eye, and demonstrates the full recovery of this
Correspondence: Sophia Bechek Stanford University School of Medicine, 29I Campus Drive, Stanford, CA 94035, USA

Tel + 650-723-686 |

Email sbechek@gmail.com 
patient following an immediate short steroid course and early introduction of enteral nutrition.

\section{Case Presentation}

A previously healthy 13-year-old boy developed a sore throat and swollen right eye two days prior to admission. His primary care physician prescribed a course of amoxicillin for pharyngitis. He subsequently developed a high fever and diarrhea, and was taken to the emergency room where a second dose of amoxicillin was administered. He defervesced and was discharged home. On the day of the admission, he was unable to tolerate oral intake, suffered two episodes of hematemesis, and developed conjunctival inflammation, blistering of his lips, and an erythematous rash on his cheeks. He was found to be tachycardic, hypertensive, and febrile at an outside emergency room and was transferred to our institution for further management.

His physical exam was notable for erythematous papules present on his trunk and extremities involving $<5 \%$ of body surface area (BSA), with diffuse erosions and sloughing of the lips, tongue, buccal and palatal mucosa. Periorbital swelling was present predominantly on the right with marked bilateral conjunctival inflammation. His abdomen was soft and non-distended with normal peristalsis. Small vesicles were noted on the scrotum without obvious involvement of urethral meatus or glans.

Within 24 hours of his admission, his diarrhea worsened and he developed mucus, sloughed mucosa and flecks of blood in his stools. Stool output was 2 to 3 liters per 24 hours while NPO. During this time, he also complained of severe abdominal cramping requiring intravenous opioids. Stool electrolytes were consistent with a secretory diarrhea. A stool polymerase chain reaction (PCR) for infectious organisms was negative for Vibrio spp., Yersinia, Salmonella, Shigella, Campylobacter, Clostridium difficile, adenovirus, norovirus, rotavirus, enterovirus, and cytomegalovirus. Serum antibody titers for Mycoplasma pneumoniae were IgG positive and IgM negative. Initial laboratory evaluation demonstrated an elevated CRP (3.2, ref. 0-0.5 mg/dL) with normal electrolytes, liver function tests and complete blood count. On hospital day 2, after the diarrhea had worsened, his labs were significant for hypoproteinemia $(5.6$, ref. $6.0-8.0 \mathrm{~g} /$ $\mathrm{dL}$ ) and hypoalbuminemia (3.1, ref. 3.5-5.0 g/dL). His hemoglobin was next evaluated on hospital day 5 and was notable for a decrease in hemoglobin (11.4, decreased from 13.7 on admission, ref. $13.0-16.0 \mathrm{~g} / \mathrm{dL})$.
He was started on methylprednisolone at $1 \mathrm{mg} / \mathrm{kg} \mathrm{IV}$ daily the morning after his admission and received aggressive IV fluid replacement for his stool losses. A 5-day course of oral azithromycin was provided given the consideration for Mycoplasma pneumoniae-induced rash and mucositis (MIRM); however, absence of respiratory symptoms, unremarkable chest imaging and negative IgM serology at time of presentation and 2 weeks later discouraged this hypothesis. A flexible fiberoptic exam revealed generalized diffuse superficial ulceration and erythema of the upper airway involving the oropharynx, aryepiglottic folds, arytenoid mucosa, and false vocal cords with mild supraglottic edema without obstruction. He did not demonstrate respiratory compromise or pooling of secretions. The patient also had severe bilateral ocular involvement requiring multiple amniotic membrane grafts.

His skin and mucosal lesions continued to evolve, with glans and urethral involvement noted on hospital day 2 and full-thickness epidermal erosions of his cheeks noted on hospital day 5 , the zenith of his skin involvement (Figure 1A).

On hospital day 4, upper endoscopy revealed severe panesophageal inflammation with denuded mucosa, proteinaceous exudates and sloughing of the mucosa with very friable tissue (Figure 1B). The stomach had minimal inflammation and no friability. Severe duodenitis, with sloughing of the mucosa and proteinaceous exudates, was also noted (Figure 1C). Flexible sigmoidoscopy revealed severe colitis with proteinaceous exudates, denuded mucosa, and membrane formation that extended to the splenic flexure (Figure 1D). Given the severity of inflammation and risk of perforation, a full colonoscopy was not performed and biopsies were not obtained. A nasogastric tube was placed under direct visualization to begin enteral feedings, and TPN was started concurrently.

His skin lesions stabilized, stool output declined, and emesis resolved by hospital day 6. Given his clinical improvement, a prednisone wean was begun. A soft, regular diet was tolerated by day 12 . By day 14 , steroids were completely discontinued and he was having formed bowel movements. He was discharged within 3 weeks of admission, and at 2 years post-discharge has not suffered any serious long-term sequelae. Unfortunately, extensive interviews with the patient and family did not reveal any new drugs, infections or exposures and the causative agent for this presentation remains unknown.

\section{Discussion}

This case demonstrates extensive involvement of the gastrointestinal tract secondary to SJS, and is the first reported 


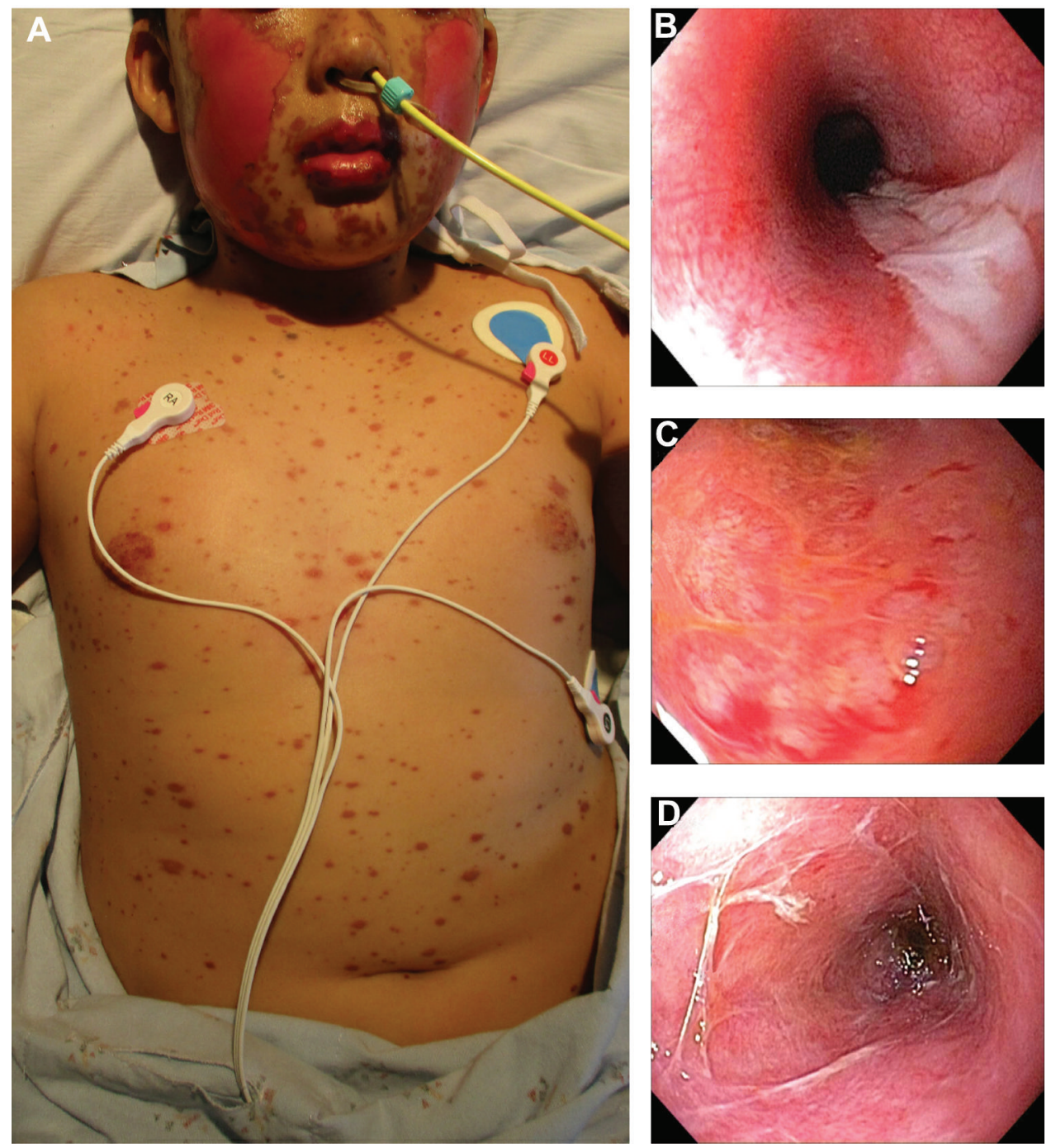

Figure I Classic erythematous papules of Stevens-Johnson syndrome present on the trunk and face with sloughing of the epidermis on the cheeks (A). Upper endoscopy images of the esophagus (B), duodenum (C), and flexible sigmoidoscopy image of the colon (D) demonstrate severe concomitant gastrointestinal involvement.

pediatric case in which both the upper and lower gastrointestinal tracts are involved. Since its first description in $1949,{ }^{16}$ we have been able to identify only 14 pediatric cases of SJS/TEN with gastrointestinal involvement, five of which had isolated esophageal involvement (Table 1). ${ }^{17-27}$ These five cases experienced the most common presentation of gastrointestinal tract involvement in children: progressive dysphagia weeks to years after the acute illness due to an esophageal stricture. ${ }^{23-27}$ Watery diarrhea with blood and mucus is the second most common presentation, either at presentation or 2-3 weeks afterwards. ${ }^{17,18,20}$ The only two pediatric cases reporting similarly profound, life-threatening diarrhea had widespread involvement of the colon and terminal ileum but did not describe disease in the upper gastrointestinal tract. One suffered recurring episodes of sepsis over 6 months and the second died of septic shock. ${ }^{17,20}$ Widespread gastrointestinal involvement is also rare in adults: two adult cases with similar involvement of the upper and lower GI tracts died from multi-organ failure within 3 weeks of presentation. ${ }^{3,5}$

In our case, the unusual and severe presentation of the disease prompted the decision to initiate steroid treatment. Dermatology consultants thought that the patient presented early enough in his disease course that a trial of steroids was warranted to try to halt disease progression. This immediate introduction of a short steroid course may have aided in the stabilization of his skin lesions and concomitant improvement of the gastrointestinal symptoms within 14 days. All but two previous pediatric cases report using steroids during the acute illness, ${ }^{17,20-26}$ and 


\begin{tabular}{|c|c|c|c|c|c|c|c|c|c|}
\hline 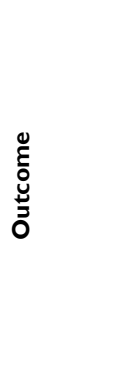 & 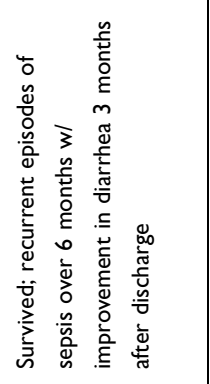 & 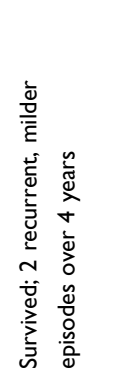 & 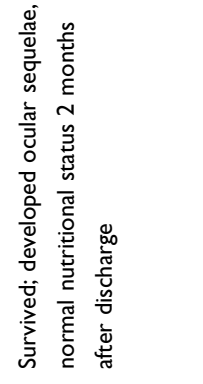 & 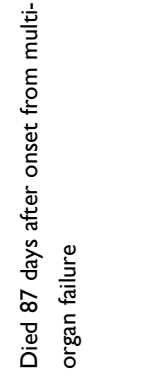 & 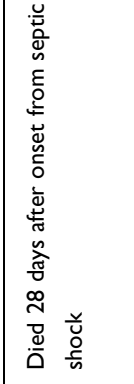 & 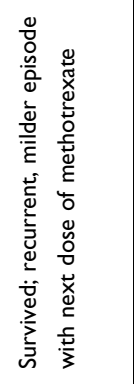 & 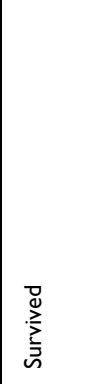 & 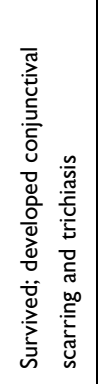 & 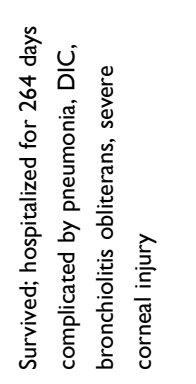 \\
\hline 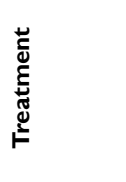 & 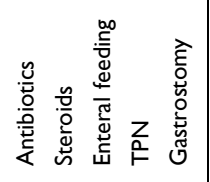 & 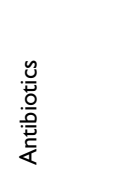 & 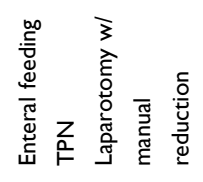 & 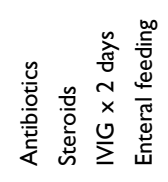 & 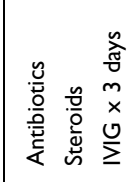 & 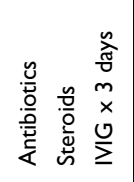 & 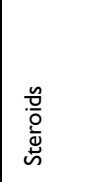 & 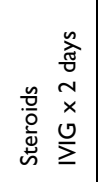 & 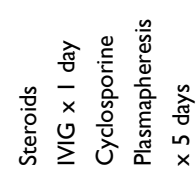 \\
\hline 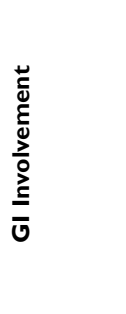 & 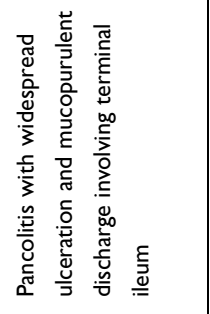 & 1 & 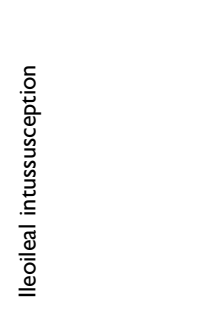 & 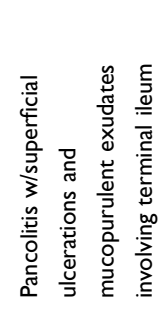 & 1 & 1 & 1 & 1 & 1 \\
\hline 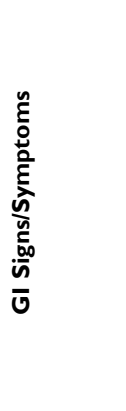 & 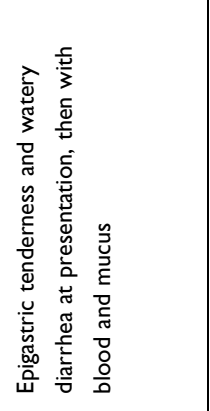 & 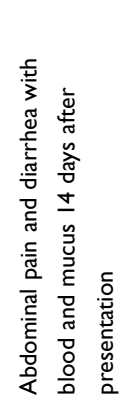 & 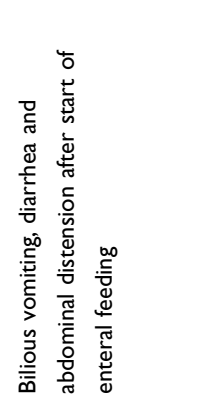 & 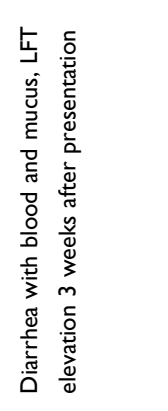 & 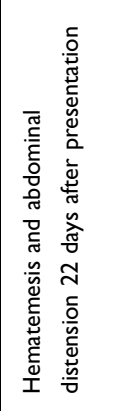 & 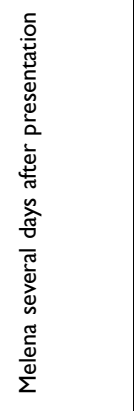 & 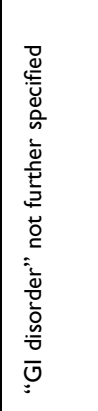 & 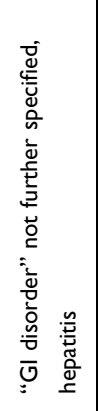 & 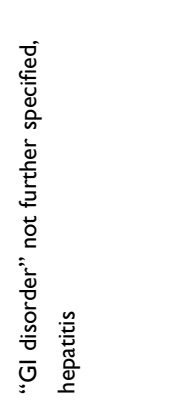 \\
\hline 台 ঐ & 1 & 1 & o & $\underline{\underline{n}}$ & $\stackrel{\sim}{\sim}$ & 음 & $\frac{O}{v}$ & ㅇ & 8 \\
\hline 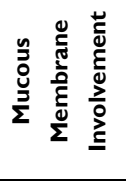 & 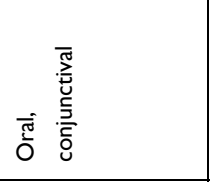 & 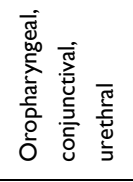 & 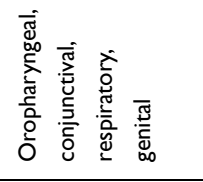 & 1 & 1 & 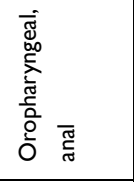 & 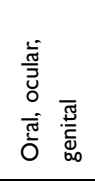 & 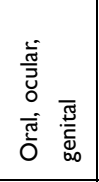 & 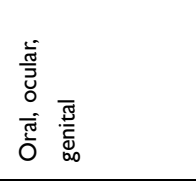 \\
\hline 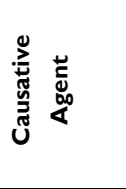 & 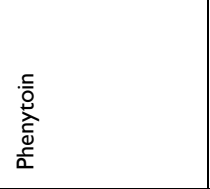 & 1 & 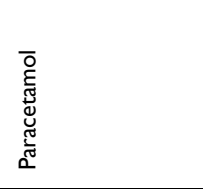 & 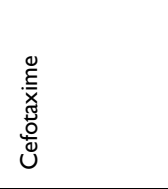 & 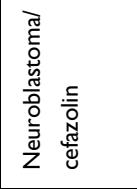 & 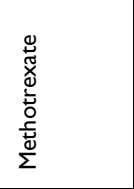 & 1 & 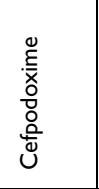 & 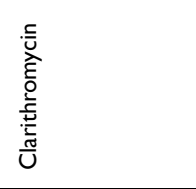 \\
\hline 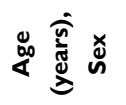 & $\begin{array}{l}\Sigma \\
\Lambda\end{array}$ & $\begin{array}{l}\Sigma \\
\underline{0}\end{array}$ & $\sum_{\infty}$ & $\stackrel{u}{i}$ & $\begin{array}{l}\Sigma \\
i\end{array}$ & $\sum_{\infty}$ & $\begin{array}{l}\Sigma \\
\text { n' }\end{array}$ & $\stackrel{u}{\sim}$ & $\stackrel{4}{=}$ \\
\hline 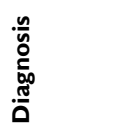 & $\cong$ & $\cong$ & $\underset{H}{\mathrm{Z}}$ & $\widetilde{n}$ & $\frac{z}{\frac{z}{5}}$ & $\widetilde{n}$ & $\cong$ & $\underset{\mathrm{H}}{\mathrm{z}}$ & 람 \\
\hline 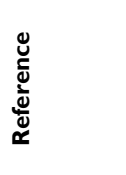 & 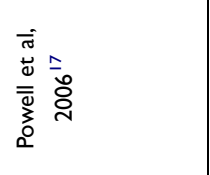 & 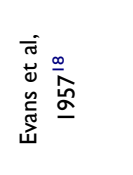 & 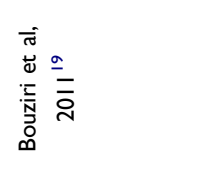 & 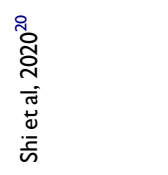 & 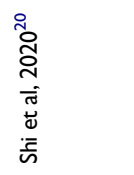 & 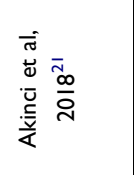 & 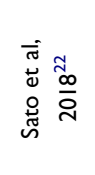 & 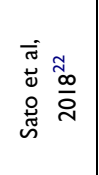 & 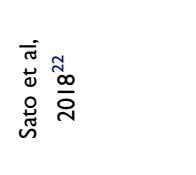 \\
\hline
\end{tabular}




\begin{tabular}{|c|c|c|c|c|}
\hline 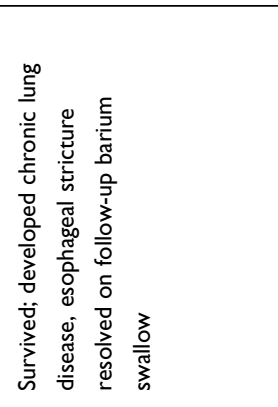 & 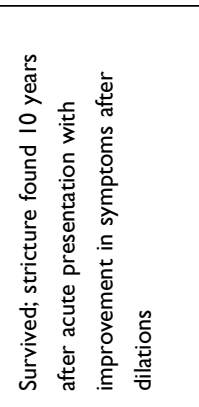 & 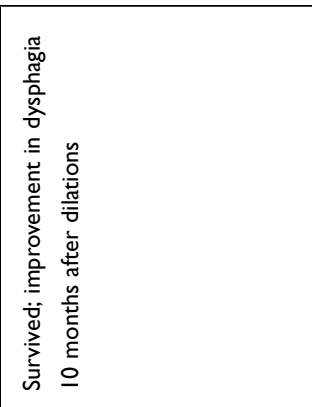 & 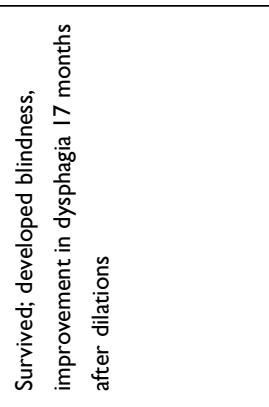 & 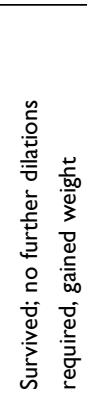 \\
\hline 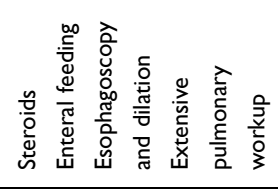 & 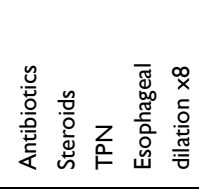 & 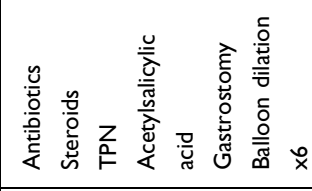 & 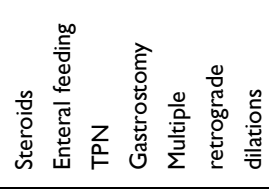 & 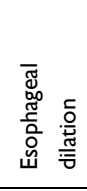 \\
\hline 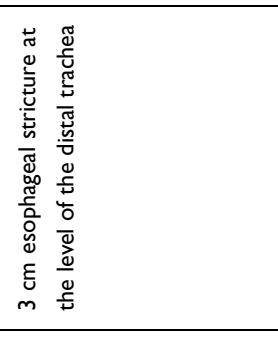 & 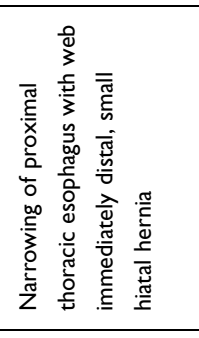 &  & 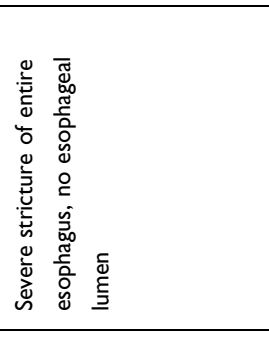 & 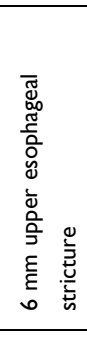 \\
\hline 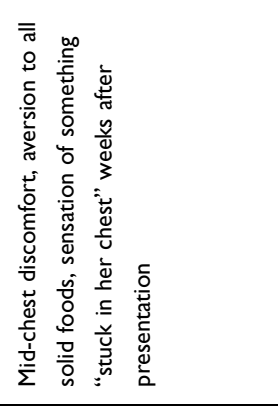 & 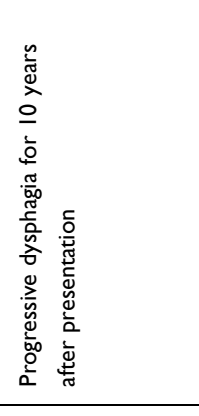 & 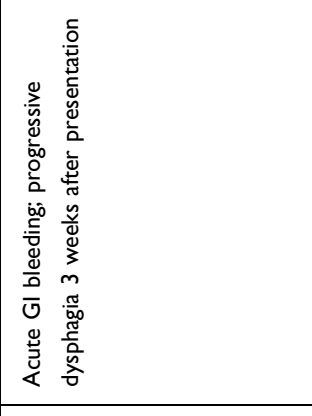 & 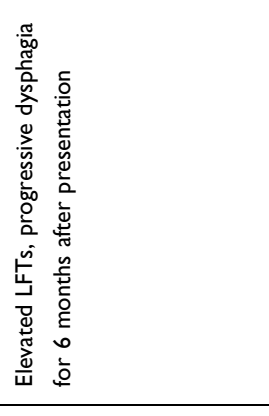 & 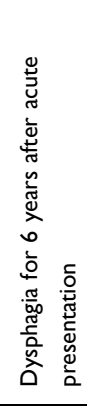 \\
\hline 1 & I & 1 & , & I \\
\hline 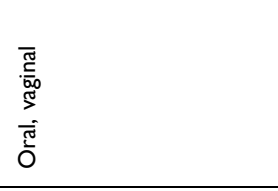 & 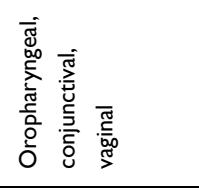 & 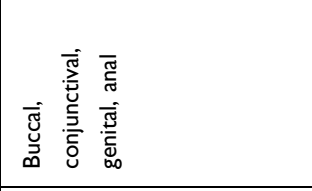 & 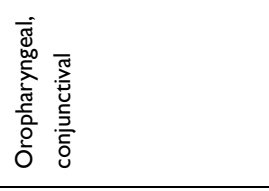 & 1 \\
\hline $\begin{array}{l}\text { 高 } \\
\overline{\bar{x}} \\
\text { 安 } \\
\end{array}$ & 1 & 1 & $\begin{array}{l}\frac{c}{\underline{\underline{z}}} \\
\text { 言 } \\
\text { 安 }\end{array}$ & 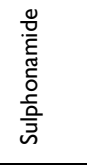 \\
\hline 峁 & $\stackrel{\stackrel{\sim}{*}}{ }$ & $\begin{array}{l}\text { L } \\
\omega^{\circ}\end{array}$ & $\Sigma$ & $\begin{array}{l}\Sigma \\
\underline{\underline{m}}\end{array}$ \\
\hline$\cong$ & 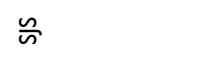 & $\cong$ & 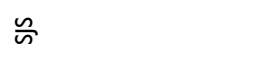 & 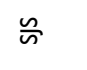 \\
\hline 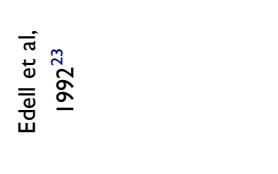 & 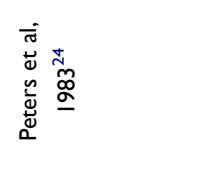 & 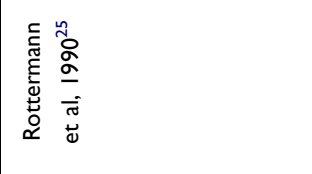 & 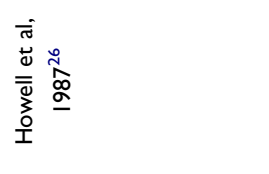 & 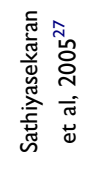 \\
\hline
\end{tabular}


five report using IVIG (Table 1). ${ }^{20-22}$ One report had no information on acute management. ${ }^{27}$ However, the usage of steroids, IVIG, or other immunosuppressive agents for the treatment of SJS/TEN remains highly controversial. ${ }^{28}$ Small prospective studies have demonstrated benefits with systemic corticosteroids, but others have shown no improvement in recovery time and a higher incidence of complications. $^{29-31}$ Similar results have confounded the use of IVIG, although evidence suggests it may be more successful in pediatric than adult patients. ${ }^{32,33}$ Any decision to initiate steroid treatment must consider possible increased mortality secondary to sepsis and the risk of bacterial translocation due to mucosal layer breakdown, as seen in previous case reports, ${ }^{17,20}$ and we believe it prudent to taper steroids when the diarrhea begins to abate and the skin lesions plateau. However, further research is clearly needed to better guide the usage of corticosteroids in the treatment of gastrointestinal manifestations of this disease.

Early initiation of an enteral diet may have also contributed to gastrointestinal recovery in our patient. Among the four previous pediatric cases of gastrointestinal tract involvement that suffered diarrhea, three were given enteral nutrition as well. ${ }^{17-20}$ While the efficacy of this practice for gastrointestinal involvement in SJS/TEN remains entirely unknown, early exposure of nutrients to a damaged bowel has been found to regenerate mucosa in other diseases involving gut mucosal repair and functional recovery. ${ }^{34,35}$ Among children with intestinal failure, early enteric feeds promote intestinal healing and regeneration even when the feeds are trophic, and absence of enteric feeding induces intestinal atrophy. ${ }^{36}$ Enteral nutrition is also thought to help during critical illness due to prevention of bacterial translocation, direct nutritional supply to rapidly proliferating mucosal epithelium and preservation of gastrointestinal motility. ${ }^{37,38}$ This literature is suggestive of the possible utility of early enteric feeds for gastrointestinal involvement of SJS/TEN, although its application is limited by the lack of studies specific to the disease.

This case highlights the need for further research to guide the usage of corticosteroids and early enteral nutrition in the treatment of gastrointestinal manifestations of this serious disease. Given the rarity of cases, multicenter investigations are likely needed to further our understanding of its management and treatment. Pediatricians should be aware of the possibility of life-threatening GI tract involvement when treating cases of SJS/TEN, and carefully consider the usage of steroids and enteric feeding in its management.

\section{Ethics Approval and Informed Consent}

The IRB at Santa Clara Valley Medical Center approved our request to submit the case report for publication. Review was conducted through an expedited review procedure.

\section{Consent for Publication}

Informed consent for publication was obtained from the patient's mother, in guidance with our institution's IRB.

\section{Author Contributions}

All authors made substantial contributions to conception and design, acquisition of data, or analysis and interpretation of data; took part in drafting the article or revising it critically for important intellectual content; agreed to submit to the current journal; gave final approval of the version to be published; and agree to be accountable for all aspects of the work.

\section{Funding}

No funding was secured for this study.

\section{Disclosure}

The authors report no conflicts of interest in this work.

\section{References}

1. Hsu DY, Brieva J, Silverberg NB, Paller AS, Silverberg JI. Pediatric Stevens-Johnson syndrome and toxic epidermal necrolysis in the United States. J Am Acad Dermatol. 2017;76(5):811-817.e814. doi:10.1016/j.jaad.2016.12.024

2. Beck MH, Portnoy B. Severe erythema multiforme complicated by fatal gastrointestinal involvement following co-trimoxazole therapy. Clin Exp Dermatol. 1979;4(2):201-204. doi:10.1111/j.13652230.1979.tb01618.x

3. Zweiban B, Cohen H, Chandrasoma P. Gastrointestinal involvement complicating Stevens-Johnson syndrome. Gastroenterology. 1986;91 (2):469-474. doi:10.1016/0016-5085(86)90585-8

4. Roupe G, Ahlmén M, Fagerberg B, Suurküla M. Toxic epidermal necrolysis with extensive mucosal erosions of the gastrointestinal and respiratory tracts. Int Arch Allergy Appl Immunol. 1986;80 (2):145-151. doi:10.1159/000234043

5. Chosidow O, Delchier JC, Chaumette MT, et al. Intestinal involvement in drug-induced toxic epidermal necrolysis. Lancet. 1991;337 (8746):928. doi:10.1016/0140-6736(91)90273-R

6. Michel P, Joly P, Ducrotte P, et al. Ileal involvement in toxic epidermal necrolysis (Lyell syndrome). Dig Dis Sci. 1993;38(10):1938-1941. doi:10.1007/BF01296123

7. Carter FM, Mitchell CK. Toxic epidermal necrolysis-an unusual cause of colonic perforation. Report of a case. Dis Colon Rectum. 1993;36 (8):773-777. doi:10.1007/BF02048370 
8. Sugimoto Y, Mizutani H, Sato T, Kawamura N, Ohkouchi K, Shimizu M. Toxic epidermal necrolysis with severe gastrointestinal mucosal cell death: a patient who excreted long tubes of dead intestinal epithelium. J Dermatol. 1998;25(8):533-538. doi:10.1111/ j.1346-8138.1998.tb02450.x

9. Sakai N, Yoshizawa Y, Amano A, et al. Toxic epidermal necrolysis complicated by multiple intestinal ulcers. Int J Dermatol. 2008;47 (2):180-182. doi:10.1111/j.1365-4632.2008.03389.x

10. Kedward AL, McKenna K. A fatal case of toxic epidermal necrolysis with extensive intestinal involvement. Clin Exp Dermatol. 2009;34 (7):e484. doi:10.1111/j.1365-2230.2009.03553.x

11. Fortinsky KJ, Fournier MR, Saloojee N. Gastrointestinal involvement in Stevens-Johnson syndrome: prompt recognition and successful treatment. Int J Colorectal Dis. 2013;28(2):285-286. doi:10.1007/ s00384-012-1483-x

12. Heye P, Descloux A, Singer G, Rosenberg R, Kocher T. Perforated sigmoid diverticulitis in the presence of toxic epidermal necrolysis. Case Rep Dermatol. 2014;6(1):49-53. doi:10.1159/000360129

13. Majima Y, Ikeda Y, Yagi H, Enokida K, Miura T, Tokura Y. Colonic involvement in Stevens-Johnson syndrome-like mucositis without skin lesions. Allergol Int. 2015;64(1):106-108. doi:10.1016/j. alit.2014.08.010

14. Claytor JD, Herfarth $\mathrm{HH}$, Weaver KN. Colonic involvement of Stevens-Johnson syndrome/toxic epidermal necrolysis: a rare cause of gastrointestinal bleeding. ACG Case Rep J. 2019;6(10):e00242. doi:10.14309/crj.0000000000000242

15. Jha AK, Suchismita A, Jha RK, Raj VK. Spectrum of gastrointestinal involvement in Stevens - Johnson syndrome. World J Gastrointest Endosc. 2019;11(2):115-123. doi:10.4253/wjge.v11.i2.115

16. Dresner E. Erythema multiforme exudativum; the Stevens-Johnson syndrome. Lancet. 1949;2(6588):1036-1038. doi:10.1016/S01406736(49)91604-9

17. Powell N, Munro JM, Rowbotham D. Colonic involvement in Stevens-Johnson syndrome. Postgrad Med J. 2006;82(968):e10. doi:10.1136/pgmj.2005.042952

18. Evans CD. Stevens-Johnson Syndrome with intestinal symptoms. $\mathrm{Br}$ J Dermatol. 1957;69(3):106-107.

19. Bouziri A, Khaldi A, Hamdi A, et al. Toxic epidermal necrolysis complicated by small bowel intussusception: a case report. J Pediatr Surg. 2011;46(2):e9-e11. doi:10.1016/j.jpedsurg.2010.09.011

20. Shi T, Chen H, Huang L, et al. Fatal pediatric Stevens-Johnson syndrome/toxic epidermal necrolysis: three case reports. Medicine. 2020;99(12):e19431. doi:10.1097/MD.0000000000019431

21. Akıncı B, Siviş Z, Şahin A, et al. Stevens-Johnson Syndrome associated with methotrexate treatment for acute lymphoblastic leukemia: a case report. Arch Argent Pediatr. 2018;116(3):e459-e462. doi:10.5546/aap.2018.eng.e459

22. Sato S, Kanbe T, Tamaki Z, et al. Clinical features of Stevens-Johnson syndrome and toxic epidermal necrolysis. Pediatr Int. 2018;60(8):697-702. doi:10.1111/ped.13613

23. Edell DS, Davidson JJ, Muelenaer AA, Majure M. Unusual manifestation of Stevens-Johnson syndrome involving the respiratory and gastrointestinal tract. Pediatrics. 1992;89(3):429-432.
24. Peters ME, Gourley G, Mann FA. Esophageal stricture and web secondary to Stevens-Johnson syndrome. Pediatr Radiol. 1983;13 (5):290-291. doi:10.1007/BF00973351

25. Rottermann EM, Julia MV, Rovira J, Pari FJ, Morales L. Esophageal stenosis following Stevens-Johnson syndrome. Treatment with balloon dilation. Clin Pediatr (Phila). 1990;29(6):336-338. doi:10.1177/ 000992289002900609

26. Howell CG, Mansberger JA, Parrish RA. Esophageal stricture secondary to Stevens-Johnson syndrome. J Pediatr Surg. 1987;22 (11):994-995. doi:10.1016/S0022-3468(87)80492-X

27. Sathiyasekaran M, Shivbalan S. Benign oesophageal stricture-a sequel of Stevens Johnson syndrome. Indian J Pediatr. 2005;72 (8):711. doi:10.1007/BF02724086

28. Liotti L, Caimmi S, Bottau P, et al. Clinical features, outcomes and treatment in children with drug induced Stevens-Johnson syndrome and toxic epidermal necrolysis. Acta Biomed. 2019;90(3-S):52-60. doi:10.23750/abm.v90i3-S.8165

29. Kakourou T, Klontza D, Soteropoulou F, Kattamis C. Corticosteroid treatment of erythema multiforme major (Stevens-Johnson syndrome) in children. Eur J Pediatr. 1997;156(2):90-93. doi:10.1007/ s004310050561

30. Rasmussen JE. Erythema multiforme in children. Response to treatment with systemic corticosteroids. Br J Dermatol. 1976;95 (2):181-186. doi:10.1111/j.1365-2133.1976.tb00824.x

31. Ting HC, Adam BA. Erythema multiforme-response to corticosteroid. Dermatologica. 1984;169(4):175-178. doi:10.1159/000249598

32. Metry DW, Jung P, Levy ML. Use of intravenous immunoglobulin in children with stevens-johnson syndrome and toxic epidermal necrolysis: seven cases and review of the literature. Pediatrics. 2003;112(6 Pt 1):1430-1436. doi:10.1542/peds.112.6.1430

33. Huang YC, Li YC, Chen TJ. The efficacy of intravenous immunoglobulin for the treatment of toxic epidermal necrolysis: a systematic review and meta-analysis. $B r J$ Dermatol. 2012;167(2):424-432. doi:10.1111/j.1365-2133.2012.10965.x

34. Ziegler TR, Evans ME, Fernández-Estívariz C, Jones DP. Trophic and cytoprotective nutrition for intestinal adaptation, mucosal repair, and barrier function. Annu Rev Nutr. 2003;23(1):229-261. doi:10.1146/annurev.nutr.23.011702.073036

35. Hansen T, Duerksen DR. Enteral nutrition in the management of pediatric and adult Crohn's disease. Nutrients. 2018;10(5):5. doi:10.3390/nu10050537

36. Gosselin KB, Duggan C. Enteral nutrition in the management of pediatric intestinal failure. $J$ Pediatr. 2014;165(6):1085-1090. doi:10.1016/j.jpeds.2014.08.012

37. Deitch EA. Bacterial translocation: the influence of dietary variables. Gut. 1994;35(1 Suppl):S23-S27. doi:10.1136/gut.35.1_Suppl.S23

38. Schörghuber M, Fruhwald S. Effects of enteral nutrition on gastrointestinal function in patients who are critically ill. Lancet Gastroenterol Hepatol. 2018;3(4):281-287. doi:10.1016/S24681253(18)30036-0
Clinical and Experimental Gastroenterology

\section{Publish your work in this journal}

Clinical and Experimental Gastroenterology is an international, peerreviewed, open access, online journal publishing original research, reports, editorials, reviews and commentaries on all aspects of gastroenterology in the clinic and laboratory. This journal is indexed on American Chemical Society's Chemical Abstracts Service (CAS)
The manuscript management system is completely online and includes a very quick and fair peer-review system, which is all easy to use. Visit http://www.dovepress.com/testimonials.php to read real quotes from published authors. 